\title{
Pembrolizumab in endometrial cancer: Where we stand now (Review)
}

\author{
AIKATERINI ARAVANTINOU-FATOROU ${ }^{1}$, ANGELIKI ANDRIKOPOULOU ${ }^{2}$, MICHAEL LIONTOS $^{2}$, \\ ORAIANTHI FISTE ${ }^{2}$, VASILIKI E. GEORGAKOPOULOU ${ }^{1}$, MELETIOS-ATHANASIOS DIMOPOULOS ${ }^{2}$, \\ MARIA GAVRIATOPOULOU ${ }^{2}$ and FLORA ZAGOURI ${ }^{2}$ \\ ${ }^{1}$ First Department of Medicine, National and Kapodistrian University of Athens, School of Medicine, \\ Laiko General Hospital, Athens 11527; ${ }^{2}$ Department of Clinical Therapeutics, Alexandra Hospital, \\ School of Medicine, National and Kapodistrian University of Athens, Athens 11528, Greece
}

Received December 24, 2020; Accepted July 2, 2021

DOI: 10.3892/ol.2021.13082

\begin{abstract}
Recently, immunotherapy has shown promising results in solid tumors. To the best of our knowledge, this is the first systematic review of published literature synthesizing all the available data and evaluating both the efficacy and safety of pembrolizumab in endometrial cancer. The present study was performed in accordance with the PRISMA guidelines. Eligible articles were identified by searching the MEDLINE and ClinicalTrials.gov databases, using a predefined combination of the terms 'endometrial cancer' and 'pembrolizumab'. Overall, nine articles incorporating data from 712 patients were eligible. Pembrolizumab was demonstrated to be an effective and safe therapeutic option for the management of advanced/metastatic endometrial cancer. Results of ongoing trials evaluating either pembrolizumab alone or in combination with other antineoplastic regimens are expected to confirm its efficacy in this setting of patients. Pembrolizumab appears to be both durable and robust in endometrial cancer. However, there is an emerging need for novel predictive biomarkers to guide clinical practice.
\end{abstract}

\section{Contents}

1. Introduction

2. Sources and study selection

3. Data on efficacy and safety

4. Discussion

5. Conclusion

Correspondence to: Professor Flora Zagouri, Department of Clinical Therapeutics, Alexandra Hospital, School of Medicine, National and Kapodistrian University of Athens, 80 Vasilissis Sofias Avenue, Athens 11528, Greece

E-mail: florazagouri@yahoo.co.uk

Key words: endometrial cancer, anti-PD1 treatment, polymerase $\varepsilon$-mutated cancer, microsatellite instability, biomarkers

\section{Introduction}

Endometrial cancer (EC) is the most common gynecological malignancy accounting for approximately 61,880 new cases and 12,160 deaths in the United States (1). Metabolic syndrome, diabetes mellitus, Lynch syndrome, hormone therapy and tamoxifen use are the main risk factors for endometrial cancer (2). Most ECs are identified at an early stage $(75 \%)$, however $25 \%$ of ECs are diagnosed at a late stage. Prognosis for advanced disease remains poor; 5 -year survival rates are $<50 \%$ for patients with lymph node involvement and $<20 \%$ for patients with peritoneal or distant metastases (3). Indeed, 5-year survival ranges from $91 \%$ for FIGO IA disease to as low as $47 \%$ for FIGO IIIC and $17 \%$ for FIGO IV disease.

Chemotherapy with carboplatin plus paclitaxel has gradually replaced the triple drug combination of cisplatin, doxorubicin and paclitaxel and is currently the standard of care in the metastatic setting $(4,5)$. Hormone treatment with progestins, anti-estrogens and aromatase inhibitors are associated with low response rates $(30 \%)(6,7)$. Nevertheless, options are limited for patients progressing on first line treatment highlighting the need for novel treatment regimens (8).

The interaction between tumorcells and T cells has been well established. Immune response is mediated by the interaction of the MHC-peptide complex with the T-cell receptor (TCR) and the binding of the co-stimulatory receptor CD28 on T cells with the ligand B7 on antigen-presenting cells (APCs) (9). In order to avoid autoimmune response cytotoxic $\mathrm{T}$ lymphocyte antigen-4 (CTLA-4) binds to CD28 and acts as a competitor for B7. Respectively, the programmed death 1 (PD-1) inhibitory receptor is expressed by T cells and binds to two ligands, namely programmed death ligand 1 (PD-L1) and programmed death ligand 2 (PD-L2) within the tumor microenvironment (10). This blockade enables the escape of tumor cells from immunosurveillance by inhibiting the activation of $\mathrm{T}$ cells and suppressing the T cell-mediated tumor cytolysis $(11,12)$. Both PD-1 and PD-L1 are targets of immunotherapeutic drugs which block their interaction and promote durable responses in a variety of cancer types $(13,14)$. 
High expression of PD-L1 seems to be a predictive biomarker for anti-PD1 therapy, although some PD-L1 negative cancers may also respond to this kind of treatment (15-17). EC cells may activate the PD-1/PD-L1 signaling pathway since they overexpress PD-L1 receptor in 25 to $100 \%$ of cases (18).

Historically, endometrial carcinoma has been classified into two main clinicopathological and molecular types: type I which includes endometrioid adenocarcinomas (80-90\%) and type II which includes non-endometrioid subtypes such as serous, clear-cell and undifferentiated carcinomas as well as carcinosarcoma (10-20\%) (2). The Cancer Genome Atlas (TCGA) Research Network proposed a different molecular classification of EC that divided EC tumors into four subgroups: i) POLE ultra-mutated, ii) microsatellite instability high (MSI-H)/mismatch repair deficient (dMMR), III) low copy number with low mutation rate, iv) high copy number with high mutation rate (19). According to TCGA Research Network some EC subtypes like POLE mutated cancers and MSI-H/dMMR groups are characterized by an immunogenic tumor microenvironment and express high level of immune checkpoint-associated proteins including PD-L1 (19).

Given the immunogenic profile of these molecular subtypes and the overexpression of PD-L1 in EC cells, monoclonal anti-PD-1 pembrolizumab could prove to be an efficient novel treatment in EC. This is the first systematic review of the literature to synthesize data from recent and ongoing clinical trials regarding the efficacy and safety of pembrolizumab in EC.

\section{Sources and study selection}

This systemic review was performed according to PRISMA guidelines (20). The protocol has been submitted to the Institutional Review Board of Alexandra University Hospital and is available upon request. Eligible articles were identified by a search of MEDLINE and Clinicaltrials.gov databases for the period up to $05 / 28 / 2021$. The search strategy included the following keywords: ((endometrial) AND (neoplasm OR cancer OR carcinoma)) AND (pembrolizumab).

English - language restrictions were applied. Two investigators Aravantinou Fatorou A (AFA) and Andrikopoulou A (AA) working independently searched the literature and extracted data from each of the eligible studies. Clinical trials were considered eligible for our study, while reviews and case reports were excluded. In order to maximize the amount of synthesized information, we systematically examined the reference lists of the articles retrieved to identify potentially eligible studies and/or conference abstracts.

Eligible articles included all studies evaluating the efficacy and safety of pembrolizumab in EC no matter of sample size. For each of these studies, the following data were collected: first author, year of publication, phase of the trial, number of patients enrolled, disease setting (metastatic etc.), overall response rate (ORR) including complete response (CR) rate and partial response (PR) (\%), stable disease (SD) rate (\%), progression disease (PD) rate (\%), median overall survival (mOS) in months, median progression free survival (mPFS) in months, data regarding biomarker analysis and adverse events. From multicohort trials, the number of patients with EC was identified and only data addressing this population were included in our study.

\section{Data on efficacy and safety}

The search strategy retrieved 72 articles. Overall, 18 reviews (21-38) and 8 case reports (39-46) were identified, while 40 studies were deemed irrelevant. After removal of ineligible studies, 6 studies were recruited (47-52). After evaluating the references of the eligible studies, three additional articles were identified (53-55). Overall, 9 studies incorporating data from 712 EC patients were eligible for this systematic review (47-55). The aforementioned process of the selection of studies is illustrated in Fig. 1. Table I presents an overview of all the ongoing or completed clinical trials with published results investigating the efficacy and safety of pembrolizumab in endometrial cancer.

Five out of nine trials were multicohort including a variety of MSI-H/ dMMR deficient tumors $(47,48,51,52,55)$ while four trials enrolled exclusively endometrial cancer patients $(49,50,53,54)$. Six clinical trials were Phase Ib/II (47,49,51-53,55), one was Phase III (54) randomized clinical trial, while two of them were retrospective, single-institution cohort studies $(48,50)$.

All patients had histologically or cytologically confirmed locally advanced, metastatic or recurrent EC. They had received at least one previous systematic treatment and had evidence of disease progression (47-55). Five studies evaluated pembrolizumab administration in patients with MSI-H/dMMR advanced EC $(48,49,52,53,55)$, one study focused on PD-L1 positive EC patients (47), while the other studies evaluated pembrolizumab administration in advanced EC irrespective of MSI or PD-L1 status $(50,51,54)$. Three studies evaluated the combination of pembrolizumab with lenvatinib in advanced/recurrent EC $(50,51,54)$ whereas the remaining studies evaluated pembrolizumab as a single agent in pretreated PD-L1 positive and/or MSI-H/dMMR advanced EC tumors (47-49,52,53,55).

664 patients received pembrolizumab $200 \mathrm{mg}$ intravenously every three weeks $(48-52,54)$ whereas 48 patients received pembrolizumab at a dose of $10 \mathrm{mg} / \mathrm{kg}$ every two weeks $(47,53,55)$. Tumor response was assessed according to the Response Evaluation Criteria in Solid Tumors (RECIST) with computer tomography (CT) or magnetic resonance (MRI) per study protocol.

Objective Response Rate (ORR) was calculated in all of the trials identified (47-55).

In PD-L1 positive advanced or recurrent EC cancer, ORR is $13 \%$ (95\% CI; 2.8-33.6\%) (NCT02054806) (47). Median PFS was 1.8 months (95\% CI; 1.6-2.7) while median OS was not reached (95\% CI; 4.3-NR).

In MSI-H/dMMR advanced EC patients with disease progression on prior standard treatment ORR was 55.9\% (57/102) $(48,49,52,53,55)$. Median PFS and OS were reported only in one study (NCT02628067) (52). Median PFS was 25.7 (95\% CI; 4.9-NR) while median OS was not reached (95\% CI; 27.2-NR). 12-month OS was reported in two studies and was $73-89 \%(52,53,56)$. Of note, $79 \%$ of patients with MSI-H/dMMR EC with change from baseline exhibited a reduction in tumor size, while in $70 \%$ of patients the reduction was greater than $30 \%(52,56)$. 


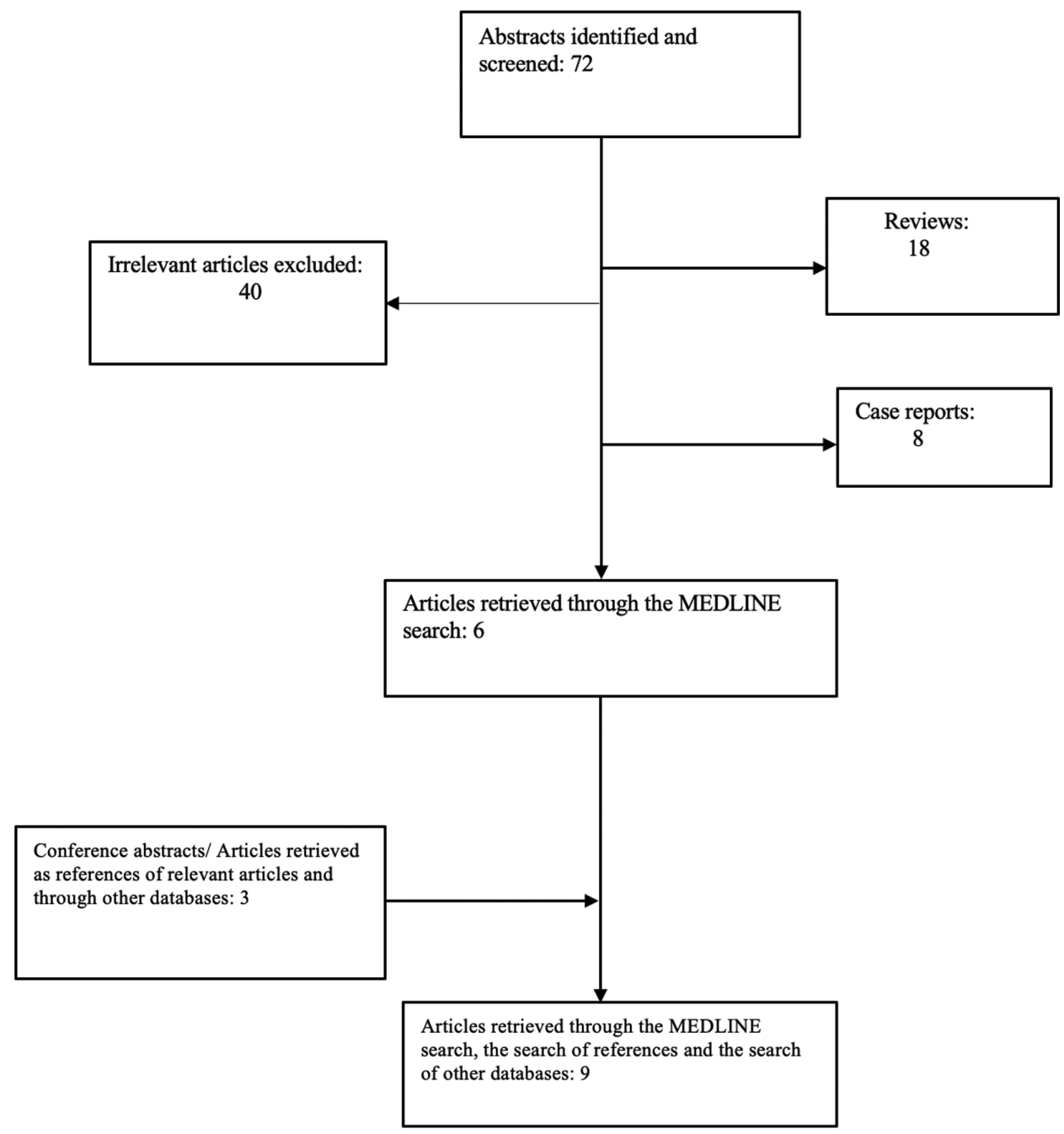

Figure 1. Stages of the search strategy.

For patients receiving the combination of pembrolizumab with lenvatinib for previously treated advanced/recurrent EC, ORR was $38.4 \%(63 / 164)$ with $6.7 \%$ of patients achieving CR $(11 / 164)$ and $31.7 \%$ of PR (52/164) $(50,51)$. In KEYNOTE-146 (NCT02501096), median PFS was 7.4 months (95\% CI; 5.3-8.7), while median OS was 16.7 months (95\% CI; 15-NR) (51). In Phase III KEYNOTE-775 trial of Lenvatinib plus pembrolizumab versus treatment of physician's choice, mPFS was 7.2 months and mOS was 18.3 months in agreement with previous results (54). Of note, ORR was higher in MSI-H/dMMR advanced EC patients. How et al (50) demonstrated that a lower starting dosage of lenvatinib than the recommended dose of $20 \mathrm{mg}$ was equally effective (response rates: 23.1 vs. $41.9 \%$; $\mathrm{P}=0.56$ ).

Safety profile. Pembrolizumab was administered as a single agent in six studies $(47-49,52,53,55)$. Safety data were reported in four of these studies [NCT02054806, NCT02628067, NCT01876511, Choi et al (48)] (47,48,52,55). Treatment-related adverse events (TRAEs) of any grade were reported in $66 \%(246 / 372)$ of patients and $15.6 \%(58 / 372)$ of patients experienced grade $3 / 4$ TRAEs. The most common treatment related AEs were fatigue $(16.9 \% ; 63 / 372)$, pruritus $(17.2 \% ; 64 / 372)$, arthralgia $(9.1 \% ; 34 / 372)$, diarrhea $(12.9 \% ; 48 / 372)$, asthenia $(7.3 \% ; 27 ; 372)$ and hypothyroidism $(11 \% ; 41 / 372)$. In all studies, there was only one case of suspected treatment-related mortality in a patient with interstitial lung disease (48).

The safety profile of pembrolizumab plus lenvatinib combination was reported in two studies (NCT02501096, NCT03517449) (51,54). In KEYNOTE-146 trial, any-grade TRAEs occurred in $96.8 \%(120 / 124)$ of patients and grade $\geq 3$ TEAEs occurred in $66.9 \%(83 / 124)$ of patients $(51)$. In KEYNOTE-775, grade $\geq 3$ TEAEs were reported in $89 \%$ of patients. Overall, 15.3-30.8\% of patients discontinued lenvatinib, 12.1-18.7\% discontinued pembrolizumab and $8.9-14 \%$ discontinued both study drugs due to treatment-related AEs. The most common TRAEs reported were: hypertension (59.7-64\%), diarrhea (52.4-54\%), hypothyroidism (43.5-57\%) and nausea (38.7-50\%). Of note, the recommended starting dose of $20 \mathrm{mg}$ lenvatinib treatment was associated with higher discontinuations (100 vs. $69.2 \%$, $\mathrm{P}=0.01)$ and treatment interruptions (93.3 vs. $73.1 \%, \mathrm{P}=0.09)$ compared with the lower starting dose of $14 \mathrm{mg}(50)$.

\section{Discussion}

Generally, pembrolizumab administered either as a single agent or in combination with other compounds is a safe and well-tolerated drug for multiple solid tumors $(57,58)$. Molecular characterization of the tumor is a prerequisite for guiding 


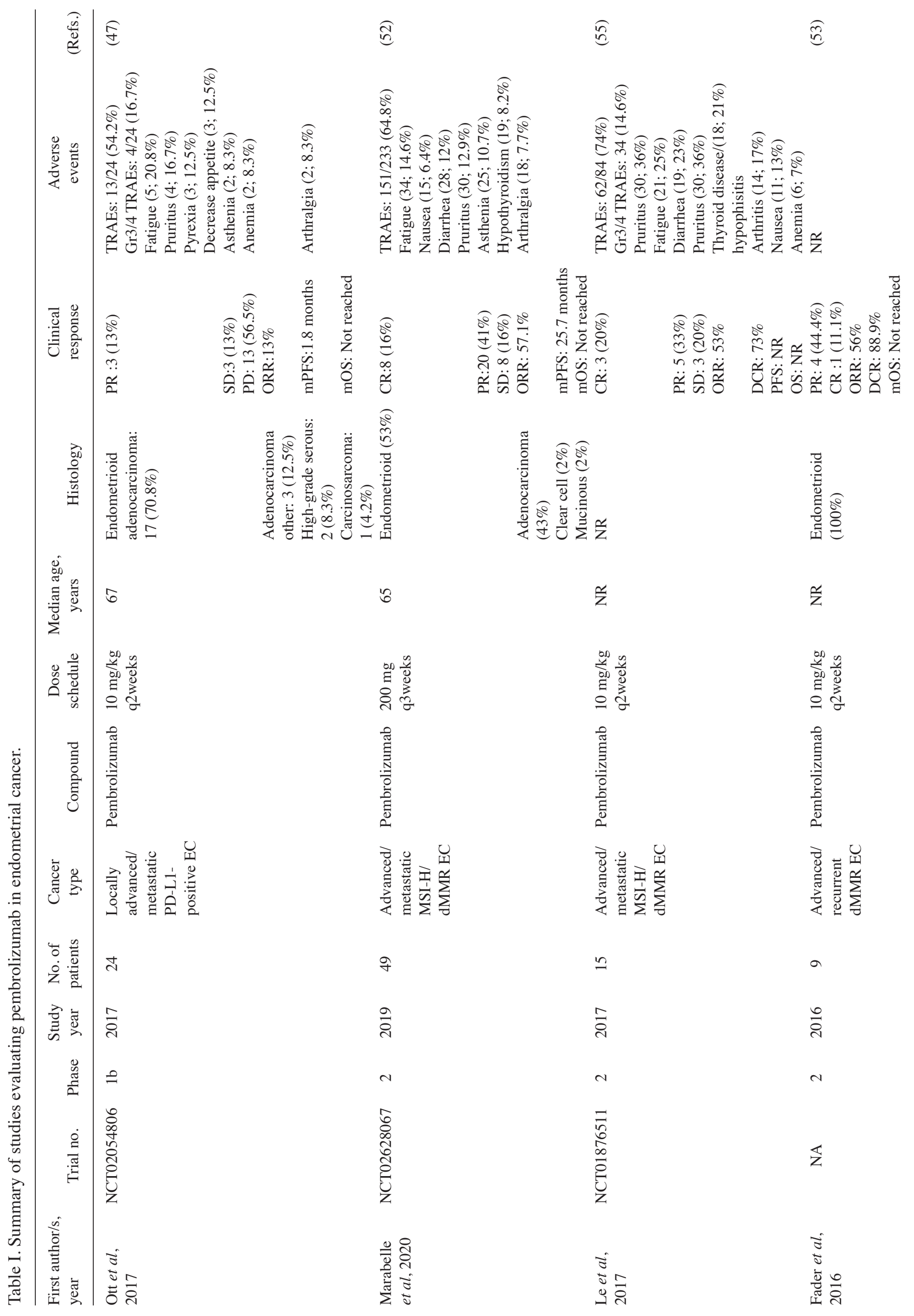




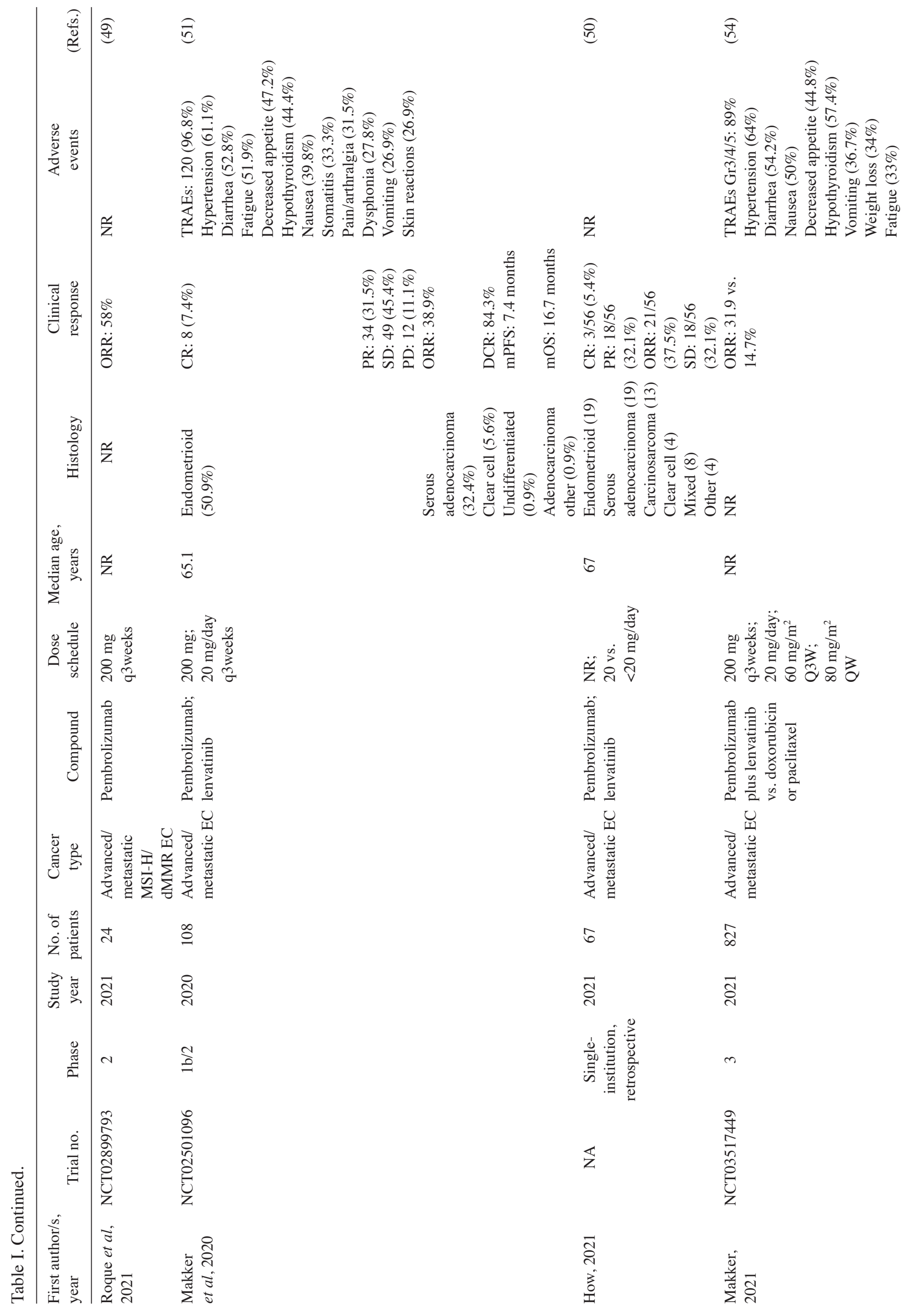




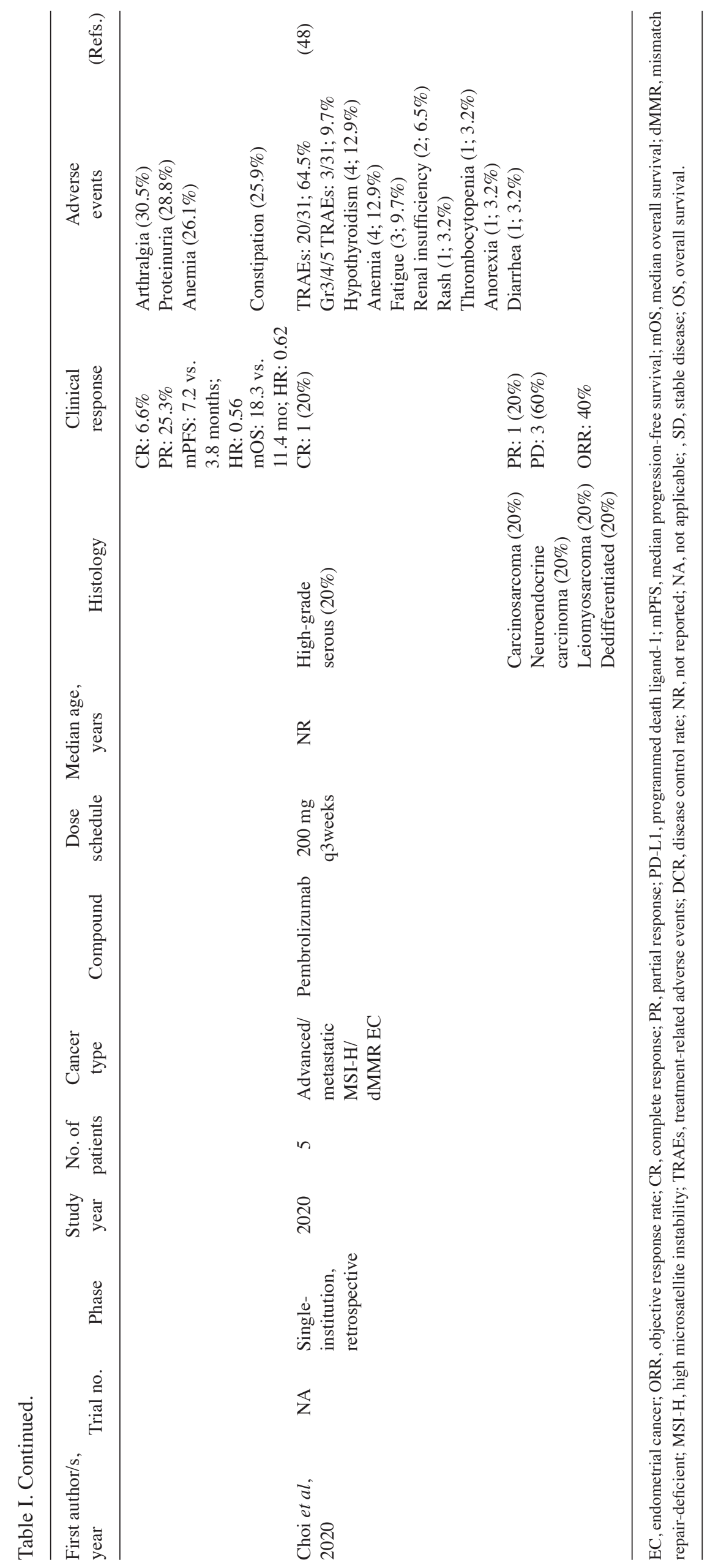




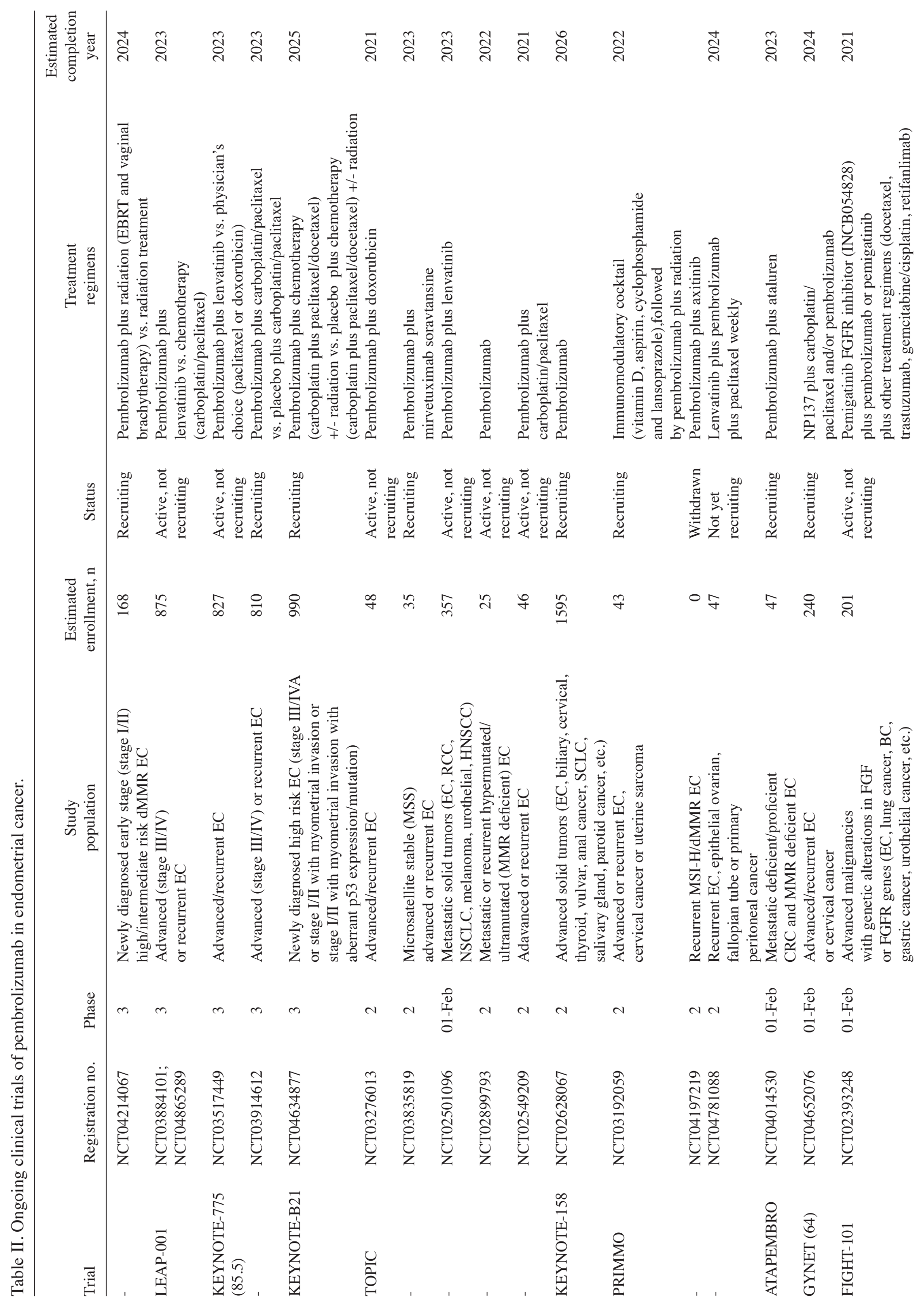




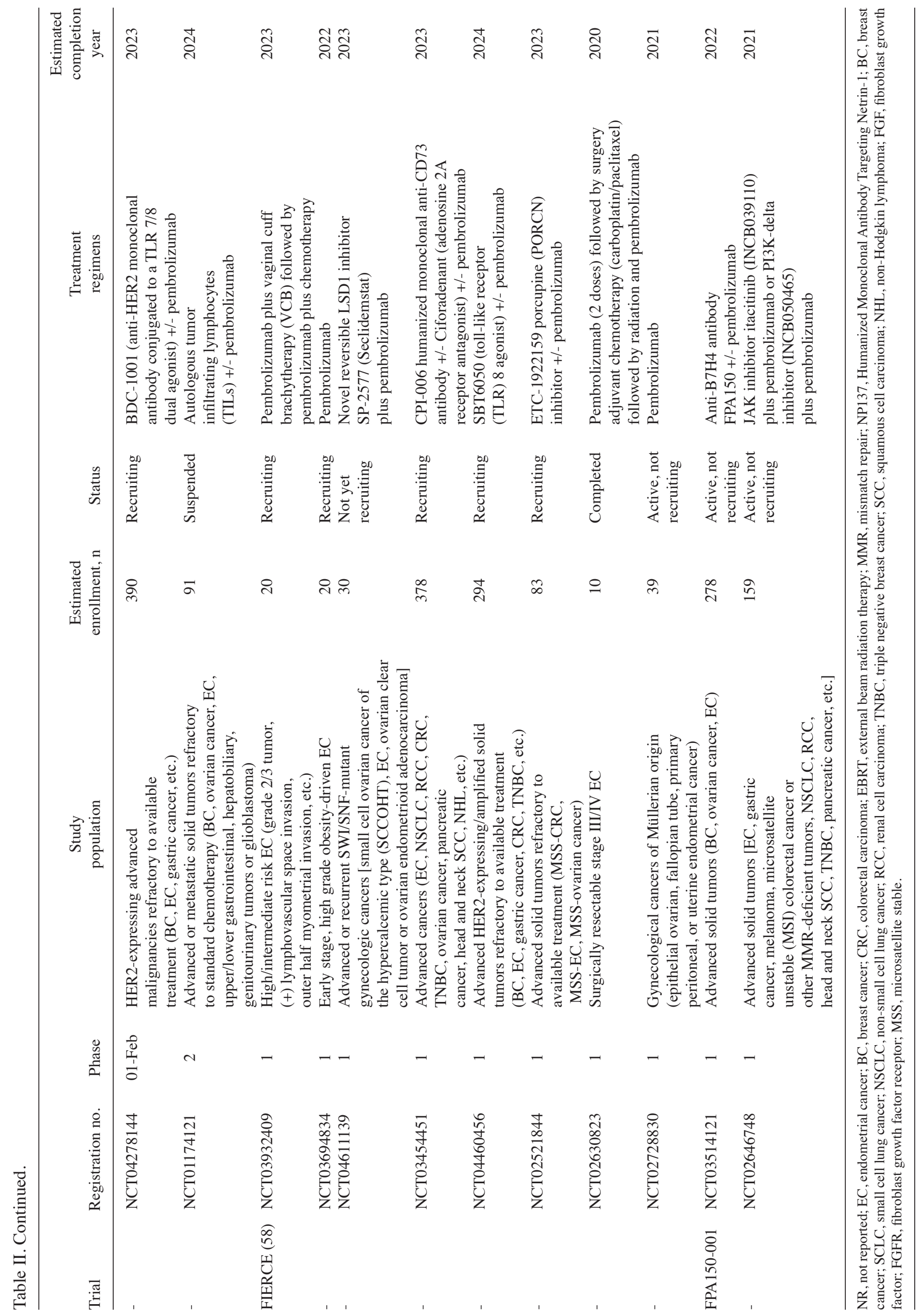


therapeutic selection of pembrolizumab in EC patients. All the ongoing clinical trials with pembrolizumab as a single agent or in combination with other treatment regimens are presented in Table II.

As already discussed, KEYNOTE-146 Phase Ib/II basket trial evaluated the combination of lenvatinib plus pembrolizumab in patients with advanced EC, regardless of PD-L1 or MSI status (51). Lenvatinib is an oral multikinase inhibitor of the vascular endothelial growth factor receptors (VEGFR)1-3, fibroblast growth factor receptors (FGFR)1-4, platelet-derived growth factor receptor- $\alpha$ (PDGFR- $\alpha$ ), RET, and KIT (59-61). On September 17, 2019 the FDA granted accelerated approval to pembrolizumab plus lenvatinib for the treatment of patients with advanced non-MSI-H/dMMR EC who exhibited disease progression to prior systemic therapy and are not candidates for curative surgery or radiation.

Two phase III, randomized, clinical trials (NCT03517449, NCT03884101) were designed to compare this combination treatment with taxane-based chemotherapy in patients with advanced EC. Final data of KEYNOTE-775 are anticipated (54). LEEP-001 (NCT03884101) Phase III trial aims to compare the efficacy of pembrolizumab plus lenvatinib to standard chemotherapy (carboplatin plus paclitaxel) for the first-line treatment of advanced (Stage III/IV) or recurrent EC $(62,63)$.

Adjuvant Radiation therapy (RT) is the treatment of choice for patients with early-stage EC and high risk of recurrence (64). Irradiated tumor cells secrete various factors that stimulate the activation of T cells and thus RT and immunotherapy may have a synergistic effect $(65,66)$. A randomized Phase III study (NCT04214067) explores the addition of pembrolizumab to standard RT in patients with newly diagnosed early stage dMMR EC of high/intermediate risk (67).

PD-L1 tumor expression presents certain limitations as a predictive biomarker (68). Firstly, a standard cut off level is not yet established. Moreover, the interaction between tumor and immune cells is a dynamic process and therefore evaluating PD-L1 at a single time point may not directly corelate to response to anti-PD1 treatment (69). In addition, PD-L1 positive EC tumors showed only a modest response (13\%) to anti-PD1 treatment compared to MSI-H/dMMR tumors (55.9\%). Thus, it could not be safely speculated that patients with EC and high expression of PDL-1 will benefit more from pembrolizumab.

DNA mismatch repair (MMR) is a key DNA repair system that identifies erroneous bases during DNA replication and recombination. Microsatellites are short DNA sequences in which a motif of one to six bases is repeated (70). Potential errors to this region are repaired by DNA MMR genes. Both mismatch repair deficiency and high microsatellite instability have been proposed as predictive biomarkers of response to immune checkpoint inhibition. dMMR and MSI-H tumors comprise approximately the $30 \%$ of ECs (17-33\%) (71). Thus, the use of MMR or/and MSI testing could be of importance in identifying patients with EC who will benefit from pembrolizumab.

DNA polymerase epsilon is a member of the DNA polymerase family that catalyzes the synthesis of DNA molecules and is essential for ensuring the fidelity of DNA replication (72). Mutations in POLE genes have been associated with high tumor mutational burden, elevated number of tumor-infiltrating and peritumoral lymphocytes, higher expression of
PD-1 and PD-L1 (73) and consequently higher response rates to immunotherapy $(72,74)$. POLE-mutated EC occurs in approximately $10 \%$ of EC cases (19). Ultra-mutated POLE ECs represent the $6.4 \%$ of low-grade and $17.4 \%$ of high-grade endometrioid tumors. Evaluation of POLE mutations may serve as an attractive predictive biomarker of susceptibility to immunotherapy (75).

Tumor mutational burden (TMB) is defined as the total number of somatic mutations per coding area. High TMB of at least 10 mutations per megabase was identified as a biomarker of favorable prognosis in KEYNOTE-158 study (76). ORR was achieved in 30 (29\%; 95\% CI: 21-39) of 102 patients in the TMB-high group versus 43 (6\%; 95\% CI: 5-8) of 688 participants in the non-TMB-high group, while duration of response was also longer in the first subgroup (not reached versus 33.1 months) (76). Overall, TMB could also serve as a biomarker of response to immune checkpoint treatment. All these potential biomarkers should be evaluated in large clinical trials before any conclusion is drawn.

To the best of our knowledge, this is the first systematic review evaluating the clinical role of pembrolizumab in EC patients. However, our study is characterized by certain limitations which should be highlighted. Eligible clinical trials were single arm, non-randomized, early Phase trials in the majority of cases $(47-53,55)$.

\section{Conclusion}

Targeting PD-1 with pembrolizumab in pretreated patients with advanced or recurrent EC represents a promising therapeutic approach and has thus received two FDA approvals as a single agent and in combination with lenvatinib. Given the profound benefit of dMMR/MSI-H patients from immunotherapy, all patients should be tested for predictive biomarkers including MMR deficiency, MSI or PD-L1 expression to guide treatment selection. Ongoing randomized, Phase III clinical trials are warranted to confirm the clinical benefit of pembrolizumab in EC patients.

\section{Acknowledgements}

The authors would like to thank Miss Fotiou Despina (Department of Clinical Therapeutics, Alexandra Hospital, National and Kapodistrian University of Athens, Athens 11528, Greece) who revised the language in the original version of the manuscript.

\section{Funding}

No funding was received.

\section{Availability of data and materials}

The datasets generated and/or analyzed during the current study are available in the Pubmed MEDLINE (https://pubmed.ncbi. nlm.nih.gov) and ClinicalTrials.gov databases (https://clinicaltrials.gov/ct2/home).

\section{Authors' contributions}

AAF wrote the manuscript and searched the literature. AA, OF and VEG searched the literature. FZ conceived the idea and 
critically revised the manuscript and gave the final approval. ML, MG and MAD critically revised the manuscript and gave the final approval. Data authentication is not applicable. All authors read and approved the final manuscript.

\section{Ethics approval and consent to participate}

Not applicable.

\section{Patient consent for publication}

Not applicable.

\section{Competing interests}

FZ has received honoraria for lectures and has served in an advisory role for Astra-Zeneca, Daiichi, Eli-Lilly, Merck, Novartis, Pfizer and Roche. ML has received honoraria from Roche, Astra Zeneca, Astellas, MSD, Janssen, BMS and IPSEN. MG has received honoraria from Janssen, Genesis Pharm, Amgen, Karyopharm and Takeda. MAD has received honoraria and has served on advisory boards from Jannsen, Genesis Pharm, Amgen, Karyopharm and Takeda, outside the submitted work. AAF, AA, VEG and OF declare that they have no competing interests.

\section{References}

1. Siegel RL, Miller KD and Jemal A: Cancer statistics, 2019. CA Cancer J Clin 69: 7-34, 2019.

2. Colombo N,Creutzberg C, Amant F, Bosse T, González-Martín A, Ledermann J, Marth C, Nout R, Querleu D, Mirza MR, et al; ESMO-ESGO-ESTRO Endometrial Consensus Conference Working Group: ESMO-ESGO-ESTRO Consensus Conference on Endometrial Cancer: Diagnosis, treatment and follow-up. Ann Oncol 27: 16-41, 2016.

3. Dowdy SC: Improving oncologic outcomes for women with endometrial cancer: Realigning our sights. Gynecol Oncol 133: 370-374, 2014.

4. Miller D, Filiaci V, Fleming G, Mannel R, Cohn D, Matsumoto T, Tewari K, DiSilvestro P, Pearl M and Zaino R: Late-Breaking Abstract 1: Randomized phase III noninferiority trial of first line chemotherapy for metastatic or recurrent endometrial carcinoma: A Gynecologic Oncology Group study. Gynecol Oncol 125: 771, 2012

5. Fleming GF: Systemic chemotherapy for uterine carcinoma: Metastatic and adjuvant. J Clin Oncol 25: 2983-2990, 2007.

6. Morice P, Leary A, Creutzberg C, Abu-Rustum N and Darai E: Endometrial Cancer 387: 1094-1108, 2016.

7. Markman M: Hormonal therapy of endometrial cancer. Eur J Cancer 41: 673-675, 2005.

8. Moxley KM and McMeekin DS: Endometrial carcinoma: A review of chemotherapy, drug resistance, and the search for new agents. Oncologist 15: 1026-1033, 2010.

9. Bourla $A B$ and Zamarin D: Immunotherapy: New strategies for the treatment of gynecologic malignancies. Oncology (Williston Park) 30: 59-66, 69, 2016

10. Pardoll DM: The blockade of immune checkpoints in cancer immunotherapy. Nat Rev Cancer 12: 252-264, 2012.

11. Sun Z, Fourcade J, Pagliano O, Chauvin JM, Sander C, Kirkwood JM and Zarour HM: IL10 and PD-1 cooperate to limit the activity of tumor-specific $\mathrm{CD}^{+} \mathrm{T}$ cells. Cancer Res 75 : $1635-1644,2015$

12. Taube JM, Anders RA, Young GD, Xu H, Sharma R, McMiller TL, Chen S, Klein AP, Pardoll DM, Topalian SL, et al: Colocalization of inflammatory response with B7-h1 expression in human melanocytic lesions supports an adaptive resistance mechanism of immune escape. Sci Transl Med 4: 127ra37, 2012.

13. Alsaab HO, Sau S, Alzhrani R, Tatiparti K, Bhise K, Kashaw SK and Iyer AK: PD-1 and PD-L1 checkpoint signaling inhibition for cancer immunotherapy: Mechanism, combinations, and clinical outcome. Front Pharmacol 8: 561, 2017.
14. Brahmer JR, Tykodi SS, Chow LQM, Hwu WJ, Topalian SL, Hwu P, Drake CG, Camacho LH, Kauh J, Odunsi K, et al: Safety and activity of anti-PD-L1 antibody in patients with advanced cancer. N Engl J Med 366: 2455-2465, 2012.

15. Ansell SM, Lesokhin AM, Borrello I, Halwani A, Scott EC, Gutierrez M, Schuster SJ, Millenson MM, Cattry D, Freeman GJ, et al: PD-1 blockade with nivolumab in relapsed or refractory Hodgkin's lymphoma. N Engl J Med 372: 311-319, 2015.

16. Larkin J, Chiarion-Sileni V, Gonzalez R, Grob JJ, Cowey CL, Lao CD, Schadendorf D, Dummer R, Smylie M, Rutkowski $\mathrm{P}$, et al: Combined nivolumab and ipilimumab or monotherapy in untreated melanoma. N Engl J Med 373: 23-34, 2015.

17. Topalian SL, Sznol M, McDermott DF, Kluger HM, Carvajal RD, Sharfman WH, Brahmer JR, Lawrence DP, Atkins MB, Powderly JD, et al: Survival, durable tumor remission, and long-term safety in patients with advanced melanoma receiving nivolumab. J Clin Oncol 32: 1020-1030, 2014

18. Herzog TJ, Arguello D, Reddy SK and Gatalica Z: PD-1, PD-L1 expression in 1599 gynecological cancers: Implications for immunotherapy. Gynecol Oncol 137: 204-205, 2015.

19. Kandoth C, Schultz N, Cherniack AD, Akbani R, Liu Y, Shen H, Robertson AG, Pashtan I, Shen R, Benz CC, et al; Cancer Genome Atlas Research Network: Integrated genomic characterization of endometrial carcinoma. Nature 497: 67-73, 2013.

20. Liberati A, Altman DG, Tetzlaff J, Mulrow C, Gøtzsche PC, Ioannidis JP, Clarke M, Devereaux PJ, Kleijnen J and Moher D: The PRISMA statement for reporting systematic reviews and meta-analyses of studies that evaluate health care interventions: explanation and elaboration. In: Journal of clinical epidemiology. J Clin Epidemiol s62: e1-34, 2009.

21. Therkildsen C, Jensen LH, Rasmussen M and Bernstein I: An update on immune checkpoint therapy for the treatment of lynch syndrome. Clin Exp Gastroenterol 14: 181-197, 2021.

22. Gómez-Raposo C, Merino Salvador M, Aguayo Zamora C, García de Santiago B and Casado Sáenz E: Immune checkpoint inhibitors in endometrial cancer. Crit Rev Oncol Hematol 161: 103306, 2021

23. Mo DC, Luo PH, Huang SX, Wang HL and Huang JF: Safety and efficacy of pembrolizumab plus lenvatinib versus pembrolizumab and lenvatinib monotherapies in cancers: A systematic review. Int Immunopharmacol 91: 107281, 2021.

24. Oaknin A, León-Castillo A and Lorusso D: Progress in the management of endometrial cancer (subtypes, immunotherapy, alterations in PIK3CA pathway): Data and perspectives. Curr Opin Oncol 32: 471-480, 2020.

25. Musacchio L, Boccia SM, Caruso G, Santangelo G, Fischetti M, Tomao F, Perniola G, Palaia I, Muzii L, Pignata S, et al: Immune checkpoint inhibitors: A promising choice for endometrial cancer patients? J Clin Med 9: 1721, 2020.

26. Green AK, Feinberg J and Makker V: A Review of immune checkpoint blockade therapy in endometrial cancer. Am Soc Clin Oncol Educ Book 40: 1-7, 2020.

27. Rubinstein MM and Makker V: Optimizing immunotherapy for gynecologic cancers. Curr Opin Obstet Gynecol 32: 1-8, 2020.

28. Sobecki-Rausch J and Barroilhet L: Anti-programmed Death-1 immunotherapy for endometrial cancer with microsatellite instability-high tumors. Curr Treat Options Oncol 20: 83, 2019.

29. Grywalska E, Sobstyl M, Putowski L and Roliński J: Current possibilities of gynecologic cancer treatment with the use of immune checkpoint inhibitors. Int J Mol Sci 20: 20, 2019.

30. Brooks RA, Fleming GF, Lastra RR, Lee NK, Moroney JW, Son $\mathrm{CH}$, Tatebe $\mathrm{K}$ and Veneris JL: Current recommendations and recent progress in endometrial cancer. CA Cancer J Clin 69: 258-279, 2019.

31. Pan K, Gong J, Huynh K and Cristea M: Current systemic treatment landscape of advanced gynecologic malignancies. Target Oncol 14: 269-283, 2019.

32. Barrington DA, Dilley SE, Smith HJ and Straughn JM Jr: Pembrolizumab in advanced recurrent endometrial cancer: A cost-effectiveness analysis. Gynecol Oncol 153: 381-384, 2019.

33. Richardson DL: New and novel therapies for gynecologic cancers. Semin Oncol Nurs 35: 217-219, 2019.

34. Garcia C and Ring KL: The role of PD-1 checkpoint inhibition in gynecologic malignancies. Curr Treat Options Oncol 19: 70, 2018.

35. Arend RC, Jones BA, Martinez A and Goodfellow P: Endometrial cancer: Molecular markers and management of advanced stage disease. Gynecol Oncol 150: 569-580, 2018. 
36. Makker V, Green AK, Wenham RM, Mutch D, Davidson B and Miller DS: New therapies for advanced, recurrent, and metastatic endometrial cancers. Gynecol Oncol Res Pract 4: 19, 2017.

37. Mittica G, Ghisoni E, Giannone G, Aglietta M, Genta S and Valabrega G: Checkpoint inhibitors in endometrial cancer: Preclinical rationale and clinical activity. Oncotarget 8: 90532-90544, 2017.

38. Tomao F, Panici PB and Tomao S: Pembrolizumab in programmed death ligand 1-positive endometrial cancer. J Clin Oncol 35: 3633, 2017.

39. Danley K, Schmitz K, Ghai R, Sclamberg JS, Buckingham LE, Burgess K, Kuzel TM and Usha L: A durable response to pembrolizumab in a patient with uterine serous carcinoma and lynch syndrome due to the MSH6 germline mutation. Oncologist: May 20, 2021 (Epub ahead of print). doi: 10.1002/ onco.13832.

40. Carvalho JP, Del Giglio A, Achatz MI and Carvalho FM: Complete clinical response in stage IVB endometrioid endometrial carcinoma after first-line pembrolizumab therapy: Report of a case with isolated loss of PMS2 protein. Case Rep Oncol 13: 1067-1074, 2020.

41. Ramos A, Del Carmen M and Yeku O: PD-1 inhibitor therapy in a patient with preexisting P-ANCA vasculitis: A case report and review of the literature. Case Rep Oncol Med 2020: 3428945, 2020.

42. Chan JK, Lakomy DS, McDonald Y and Kapp DS: Long-term durable responses after pembrolizumab immunotherapy for recurrent, resistant endometrial cancer. Gynecol Oncol Rep 33: $100581,2020$.

43. Takeda A, Koike W and Watanabe K: Rapid regression of microsatellite instability-high/programmed cell death ligand 1-negative recurrent endometrial carcinoma by immune checkpoint blockade with pembrolizumab: A case report and literature review. Gynecol Oncol Rep 32: 100553, 2020.

44. Veneris JT, Lee EK, Goebel EA, Nucci MR, Lindeman N, Horowitz NS, Lee L, Raut CP, Crotzer D, Matulonis U, et al: Diagnosis and management of a recurrent polymerase-epsilon (POLE)-mutated endometrial cancer. Gynecol Oncol 153: 471-478, 2019

45. Dizon DS, Dias-Santagata D, Bregar A, Sullivan L, Filipi J, DiTavi E, Miller L, Ellisen L, Birrer M and DelCarmen M: Complete remission following pembrolizumab in a woman with mismatch repair-deficient endometrial cancer and a germline BRCA1 mutation. Oncologist 23: 650-653, 2018.

46. Mehnert JM, Panda A, Zhong H, Hirshfield K, Damare S, Lane K, Sokol L, Stein MN, Rodriguez-Rodriquez L, Kaufman HL, et al: Immune activation and response to pembrolizumab in POLE-mutant endometrial cancer. J Clin Invest 126: 2334-2340, 2016.

47. Ott PA, Bang YJ, Berton-Rigaud D, Elez E, Pishvaian MJ, Rugo HS, Puzanov I, Mehnert JM, Aung KL, Lopez J, et al: Safety and antitumor activity of pembrolizumab in advanced programmed death ligand 1-positive endometrial cancer: Results from the KEYNOTE-028 study. J Clin Oncol 35: 2535-2541, 2017.

48. Choi MC, Moon YW, Jung SG, Park H, Joo WD, Song SH, Lee C, Kim G and Kim KA: Real-world experience with pembrolizumab treatment in patients with heavily treated recurrent gynecologic malignancies. Yonsei Med J 61: 844-850, 2020.

49. Roque DM, Bellone S, Siegel ER, Buza N, Bonazzoli E, Guglielmi A, Zammataro L, Nagarkatti N, Zaidi S, Lee J, et al: A phase II evaluation of pembrolizumab in recurrent microsatellite instability-high (MSI-H) endometrial cancer patients with Lynch-like versus MLH-1 methylated characteristics (NCT02899793). J Clin Oncol 39 (Suppl 15): 5523-5523, 2021.

50. How J: The use of pembrolizumab and lenvatinib combination therapy in endometrial cancer: An examination of toxicity and treatment efficacy in clinical practice Presented at: The virtual 2021 SGO Annual Meeting on Women's Cancer; March 19-25, 2021. Abstract 10775

51. Makker V, Taylor MH, Aghajanian C, Oaknin A, Mier J, Cohn AL, Romeo M, Bratos R, Brose MS, DiSimone C, et al: Lenvatinib plus pembrolizumab in patients with advanced endometrial cancer. J Clin Oncol 38: 2981-2992, 2020.

52. Marabelle A, Le DT, Ascierto PA, Di Giacomo AM, De Jesus-Acosta A, Delord JP, Geva R, Gottfried M, Penel N, Hansen AR, et al: Efficacy of pembrolizumab in patients with noncolorectal high microsatellite instability/mismatch repair-deficient cancer: Results from the phase II KEYNOTE-158 study. J Clin Oncol 38: 1-10, 2020
53. Fader AN, Diaz LA, Armstrong DK, Tanner EJ III, Uram J, Eyring A, Wang H, Fisher G, Greten T and Le D: Preliminary results of a phase II study: PD-1 blockade in mismatch repair-deficient, recurrent or persistent endometrial cancer. Gynecol Oncol 141: 206-207, 2016.

54. Makker V: A multicenter, open-label, randomized, phases III study to compare the efficacy and safety of lenvatinib in combination with pembrolizumab versus treatment of physician's choice in patients with advanced endometrial cancer Abstract ID: 11512. Presented at the Society of Gynecologic Oncology Virtual Annual Meeting on Women's Cancer, March $19-25,2021$

55. Le DT, Durham JN, Smith KN, Wang H, Bartlett BR, Aulakh LK, Lu S, Kemberling H, Wilt C, Luber BS, et al: Mismatch repair deficiency predicts response of solid tumors to PD-1 blockade. Science 357: 409-413, 2017.

56. O'Malley D, Marabelle A, De Jesus-Acosta A, Piha-Paul SA, Arkhipov A, Longo F, Motola-Kuba D, Shapira-Frommer R, Geva R, Rimel BJ, et al: Pembrolizumab in patients with MSI-H advanced endometrial cancer from the KEYNOTE-158 study. Ann Oncol 30: v425-v426, 2019.

57. Robert C, Schachter J, Long GV, Arance A, Grob JJ, Mortier L, Daud A, Carlino MS, McNeil C, Lotem M, et al; KEYNOTE-006 investigators: Pembrolizumab versus ipilimumab in advanced melanoma. N Engl J Med 372: 2521-2532, 2015.

58. Gandhi L, Rodríguez-Abreu D, Gadgeel S, Esteban E, Felip E, De Angelis F, Domine M, Clingan P, Hochmair MJ, Powell SF, et al; KEYNOTE-189 investigators: pembrolizumab plus chemotherapy in metastatic non-small-cell lung cancer. N Engl J Med 378: 2078-2092, 2018.

59. Matsui J, Yamamoto Y, Funahashi Y, Tsuruoka A, Watanabe T, Wakabayashi T, Uenaka T and Asada M: E7080, a novel inhibitor that targets multiple kinases, has potent antitumor activities against stem cell factor producing human small cell lung cancer $\mathrm{H} 146$, based on angiogenesis inhibition. Int J Cancer 122: 664-671, 2008

60. Tohyama O, Matsui J, Kodama K, Hata-Sugi N, Kimura T, Okamoto K, Minoshima Y, Iwata M and Funahashi Y: Antitumor activity of lenvatinib (E7080): An angiogenesis inhibitor that targets multiple receptor tyrosine kinases in preclinical human thyroid cancer models. J Thyroid Res 2014: 638747, 2014.

61. Okamoto K, Kodama K, Takase K, Sugi NH, Yamamoto Y, Iwata $\mathrm{M}$ and Tsuruoka $\mathrm{A}$ : Antitumor activities of the targeted multi-tyrosine kinase inhibitor lenvatinib (E7080) against RET gene fusion-driven tumor models. Cancer Lett 340: 97-103, 2013

62. ClinicalTrials.gov: Pembrolizumab (MK-3475) Plus Lenvatinib (E7080/MK-7902) Versus Chemotherapy for Endometrial Carcinoma (ENGOT-en9/MK-7902-001) (LEAP-001). ClinicalTrials.gov Identifier: NCT03884101. https://www.clinicaltrials.gov/ct2/show/NCT03884101. Accessed June 16, 2021.

63. ClinicalTrials.gov: Pembrolizumab (MK-3475) Plus Lenvatinib (E7080/MK-7902) Versus Chemotherapy for Endometrial Carcinoma (ENGOT-en9 MK-7902-001). China Extension Study (LEAP -001). ClinicalTrials.gov Identifier: NCT04865289. https://clinicaltrials.gov/ct2/show/NCT04865289. Accessed June $16,2021$.

64. Klopp A, Smith BD, Alektiar K, Cabrera A, Damato AL, Erickson B, Fleming G, Gaffney D, Greven K, Lu K, et al: The role of postoperative radiation therapy for endometrial cancer: Executive summary of an American Society for Radiation Oncology evidence-based guideline. Pract Radiat Oncol 4: 137-144, 2014.

65. Tang C, Wang X, Soh H, Seyedin S, Cortez MA, Krishnan S, Massarelli E, Hong D, Naing A, Diab A, et al: Combining radiation and immunotherapy: A new systemic therapy for solid tumors? Cancer Immunol Res 2: 831-838, 2014.

66. Reits EA, Hodge JW, Herberts CA, Groothuis TA, Chakraborty M, Wansley EK, Camphausen K, Luiten RM, de Ru AH, Neijssen J, et al: Radiation modulates the peptide repertoire, enhances MHC class I expression, and induces successful antitumor immunotherapy. J Exp Med 203: 1259-1271, 2006.

67. ClinicalTrials.gov: Testing the Addition of the Immunotherapy Drug, Pembrolizumab, to the Usual Radiation Treatment for Newly Diagnosed Early Stage High Intermediate Risk Endometrial Cancer. ClinicalTrials.gov Identifier: NCT04214067. https://clinicaltrials.gov/ct2/show/NCT04214067. Accessed June $16,2021$.

68. Patel SP and Kurzrock R: PD-L1 expression as a predictive biomarker in cancer immunotherapy. Mol Cancer Ther 14: $847-856,2015$. 
69. Mukherji D, Jabbour MN, Saroufim M, Temraz S, Nasr R, Charafeddine M, Assi R, Shamseddine A and Tawil AN: Programmed death-ligand 1 expression in muscle-invasive bladder cancer cystectomy specimens and lymph node metastasis: A reliable treatment selection biomarker? Clin Genitourin Cancer 14: 183-187, 2016.

70. Schlötterer C: Evolutionary dynamics of microsatellite DNA. Chromosoma 109: 365-371, 2000.

71. Murali R, Soslow RA and Weigelt B: Classification of endometrial carcinoma: More than two types. Lancet Oncol 15 e268-e278, 2014.

72. Rayner E, van Gool IC, Palles C, Kearsey SE, Bosse T, Tomlinson I and Church DN: A panoply of errors: Polymerase proofreading domain mutations in cancer. Nat Rev Cancer 16: 71-81, 2016.

73. Bellone S, Centritto F, Black J, Schwab C, English D, Cocco E, Lopez S, Bonazzoli E, Predolini F, Ferrari F, et al: Polymerase $\varepsilon$ (POLE) ultra-mutated tumors induce robust tumor-specific $\mathrm{CD}^{+} \mathrm{T}$ cell responses in endometrial cancer patients. Gynecol Oncol 138: 11-17, 2015.
74. Uryvaev A, Passhak M, Hershkovits D, Sabo E and Bar-Sela G: The role of tumor-infiltrating lymphocytes (TILs) as a predictive biomarker of response to anti-PD1 therapy in patients with metastatic non-small cell lung cancer or metastatic melanoma. Med Oncol 35: 25, 2018.

75. Wang F, Zhao Q, Wang YN, Jin Y, He MM, Liu ZX and Xu RH: Evaluation of POLE and POLD1 mutations as biomarkers for immunotherapy outcomes across multiple cancer types. JAMA Oncol 5: 1504-1506, 2019.

76. Marabelle A, Fakih M, Lopez J, Shah M, Shapira-Frommer R, Nakagawa K, Chung HC, Kindler HL, Lopez-Martin JA, Miller WH Jr, et al: Association of tumour mutational burden with outcomes in patients with advanced solid tumours treated with pembrolizumab: Prospective biomarker analysis of the multicohort, open-label, phase 2 KEYNOTE-158 study. Lancet Oncol 21: 1353-1365, 2020

This work is licensed under a Creative Commons Attribution-NonCommercial-NoDerivatives 4.0 International (CC BY-NC-ND 4.0) License. 